\title{
RECOMBINATION PROCESSES IN ZnSe:Eu
}

\author{
K. ŚWIĄTEK, M. GodLEwSKI \\ Institute of Physics, Polish Academy of Sciences \\ Al. Lotników 32/46, 02-668 Warszawa, Poland
}

The photo-ESR and photoluminescence experiments have been performed on high-resistivity $\mathrm{ZnSe:Eu} \mathrm{crystals.} \mathrm{We} \mathrm{report} \mathrm{the} \mathrm{first} \mathrm{evidence} \mathrm{that} \mathrm{the}$ energy level of $\mathrm{Eu}^{2+}$ ground state is located within the $\mathrm{ZnSe}$ forbidden gap, approximately $2.1 \mathrm{eV}$ below the bottom of the conduction band.

PACS numbers: $71.55 . \mathrm{Gs}, 76.30 . \mathrm{Kg}, 78.50 . \mathrm{Ge}$

Contrary to practically all transition-metal impurities only some rare-earth (RE) ions have been assumed to introduce energy levels into the forbidden gap of II-VI compounds [1]. Recently, we have analyzed this problem in $\mathrm{ZnS}$ and other sulphides doped with RE ions [2]. Until now, the only RE impurity observed in ZnSe lattice in 2+ charge state is europium. [3]. In this communication we present the first evidence that the $\mathrm{Eu}^{2+/ 3+}$ energy level is located within the $\mathrm{ZnSe}$ gap.

The electron spin resonance (ESR) experiments were performed on a Bruker 418s X-band equipped with an Oxford Instruments ESR-900 continuous-gas-flow cryostat, working in the temperature range of 4-300 K. A high-pressure XBO 150 xenon lamp and a set of Carl-Zeiss interference filters were used for the optical excitation. The photo-ESR experiments have been performed on high-resistivity $\mathrm{ZnSe:Eu} \mathrm{crystals.} \mathrm{An} \mathrm{ESR} \mathrm{signal} \mathrm{of} \mathrm{Eu}^{2+}\left(4 f^{7}\right)$ has been observed at $4.2 \mathrm{~K}$ prior to illumination. After illuminating the sample with $h \nu_{1}>1.9 \mathrm{eV}$ light a quenching of the intensity of this signal has been observed, i.e., the concentration of $\mathrm{Eu}^{2+}$ centers was found to decrease due to the population of the $\mathrm{Eu}^{3+}\left(4 f^{6}\right)$ state which cannot be easily detected by ESR technique. Then the light was turned off and a small increase of the $\mathrm{Eu}^{2+} \mathrm{ESR}$ signal was observed. After equilibrium was reached, a secondary infrared illumination $\left(0.9 \mathrm{eV}<h \nu_{2}<1.1 \mathrm{eV}\right)$ was applied leading to rapid enhancement of the $\mathrm{Eu}^{2+}$ signal intensity. Still a small, further increase occurred after the light was turned off. The sequence of steps in the experiment is shown in Fig. 1. Before each measurement, a primary $h \nu_{2}$ illumination was applied to ensure the same initial conditions. The spectral distribution of the photoquenching rate constant $\left(\tau^{-1}\right)$ normalized to constant light intensity is shown in Fig. 2. 


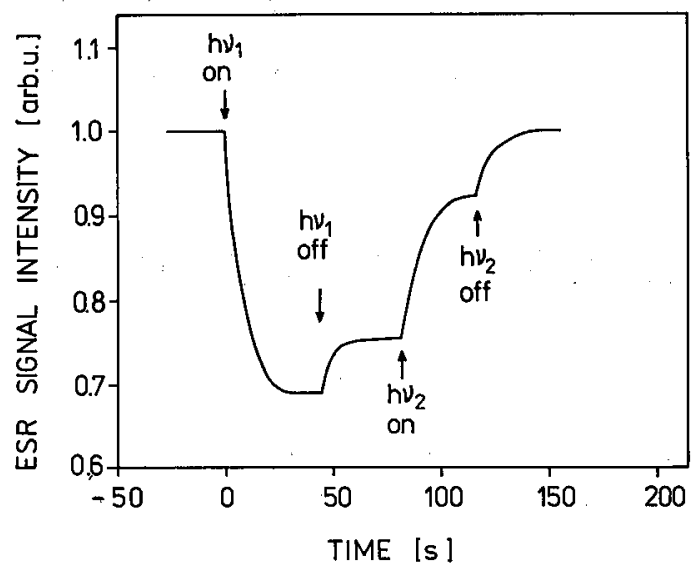

Fig. 1. The sequence of steps in the photo-ESR experiment. Kinetics of the intensity of the $\mathrm{Eu}^{2+} \mathrm{ESR}$ signal under the primary $h \nu_{1}=2.2 \mathrm{eV}$ and secondary $h \nu_{2}=1 \mathrm{eV}$ illumination are shown.

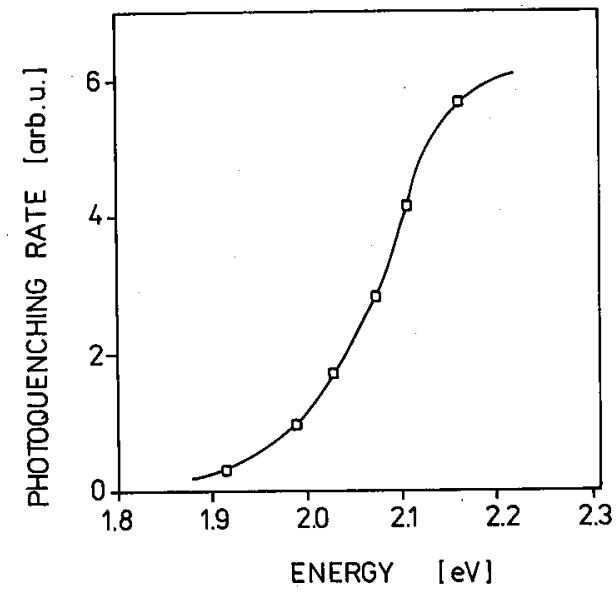

Fig. 2. The spectral dependence of the $\mathrm{Eu}^{2+}$ photoquenching rate measured at $4.2 \mathrm{~K}$. The solid curve represents the fit to the experimental data with formulas (1) and (2).

We attribute the first transition $\left(h \nu_{1}>1.9 \mathrm{eV}\right)$ to the direct $\mathrm{Eu}^{2+}+h \nu_{1} \Rightarrow$ $\mathrm{Eu}^{3+}+\mathrm{e}_{\mathrm{CB}}$ photoionization. By solving the appropriate kinetic equations [4] it can be shown that $\tau^{-1}(h \nu)$ is proportional to the optical cross-section $\sigma(h \nu)$ for this transition. Thus, the spectral dependence of the $\mathrm{Eu}^{2+}$ ionization can be determined. Since for the description of a smooth band observed in a narrow energy range an elaborate theoretical approach is not justified, we used the simple 
Kopylov-Pikhtin formula [5]:

$$
\sigma\left(E_{\text {opt }}, h \nu\right) \propto\left(h \nu-E_{\text {opt }}\right)^{1 / 2}(h \nu)^{-3} .
$$

Because in a partly ionic compound the change of a center's charge state is accompanied by a relaxation of the lattice around the ionized ion, this formula was extended to account for the electron-phonon interaction in the manner proposed by Langer et al. [6]:

$$
\begin{aligned}
& \sigma(h \nu)=\pi^{-1 / 2} \int_{-\beta}^{\infty} \mathrm{d} z \sigma_{\mathrm{el}}\left(E_{\mathrm{opt}}, h \nu+\Gamma z\right)(1+\Gamma z / h \nu) \exp \left(-z^{2}\right), \\
& \beta=\left(h \nu-E_{\mathrm{opt}}\right) / \Gamma \\
& \Gamma=\frac{\omega_{0}}{\omega_{\mathrm{ex}}}\left[2\left(E_{\mathrm{opt}}-E_{\mathrm{th}}\right) \hbar \omega_{0} \operatorname{cth}\left(\frac{\hbar \omega_{0}}{2 k T}\right)\right]^{-1 / 2} .
\end{aligned}
$$

From the fit we have obtained the thermal and optical ionization energies of $\mathrm{Eu}^{2+}$ impurity: $E_{\mathrm{th}}=1.9 \pm 0.1 \mathrm{eV}$ and $E_{\mathrm{opt}}=2.1 \pm 0.1 \mathrm{eV}$, respectively.

The complementary photoneutralization transition $\mathrm{Eu}^{3+}+h \nu_{2} \Rightarrow \mathrm{Eu}^{2+}+h_{\mathrm{VB}}$ has been observed for $0.7 \mathrm{eV} \leq h \nu_{2} \leq 1.1 \mathrm{eV}$ as an increase of the $\mathrm{Eu}^{2+} \mathrm{ESR}$ signal intensity. This part of measurements is less accurate due to competition of many extra processes leading to both stimulation and quenching of $\mathrm{Eu}^{2+}$, i.e., the capture of electrons ionized from donors into the conduction band, capture of holes generated in the valence band in the ionization process of chromium and copper-related defects ( $\mathrm{Cr}$ and $\mathrm{Cu}$ are contaminants in our samples).

The photosensitivity of the $\mathrm{Eu}^{2+}$ charge state must mean that $\mathrm{Eu}^{3+}$ is present, or can be obtained in the $\mathrm{ZnSe}$ host. The proper excitation mechanism of the $\mathrm{Eu}^{3+}$ luminescence is still a separate problem recently discussed by us for $\mathrm{ZnS}: \mathrm{Eu}$ [9]. The efficient charge transfer between $\mathrm{Eu}^{2+}$ and $\mathrm{Cr}^{+}$indicates that europium can act as a recombination center of free carriers generated in conduction and valence band of $\mathrm{ZnSe}$. In the photoluminescence experiments we observed well known emission bands associated with the presence of $\mathrm{Cu}$ impurity, namely the green $(2.34 \mathrm{eV})$, red $(1.95 \mathrm{eV})$ and infrared $(1.41 \mathrm{eV})$ emissions $[7,8]$. In these studies we have not observed any $\mathrm{Eu}^{3+}$-related intra-ion emission.

\section{References}

[1] J.W. Allen, in Electroluminescence, Springer Proceedings in Physics 38, Eds. S. Shionoya, H. Kobayashi, Springer, Berlin 1989, p. 10.

[2] K. Świątek, A. Suchocki, M. Godlewski, Appl. Phys. Lett. 56, 195 (1990).

[3] S. Ibuki, H. Komiya, M. Nakada, H. Masui, H. Kimura, J. Lumin. 1/2, 797 (1970).

[4] M. Godlewski, Phys. Status Solidi A 90, 11 (1985).

[5] A.A. Kopylov, A.N. Pikhtin, Fiz. Tverd. Tela 16, 1837 (1974).

[6] U. Piekara, J.M. Langer, B. Krukowska-Fulde, Solid State Commun. 23, 583 (1977); 
J.M. Langer, Lect. Notes Phys. 122, 123 (1980).

[7] S. Shionoya, in Luminescence of Inorganic Solids, Ed. P. Goldberg, Academic Press, New York 1966, Chapt. 2, p. 206.

[8] M. Godlewski, W.E. Lamb, B.C. Cavenett, Solid State Commun. 39, 595 (1981).

[9] K. Świątek, M. Godlewski, D. Hommel, Phys. Rev. B 42, 3628 (1990). 